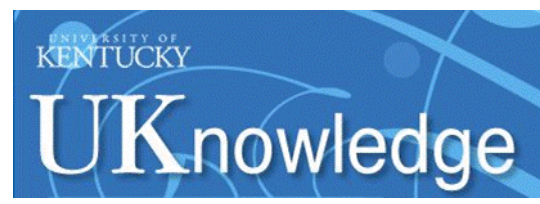

University of Kentucky

UKnowledge

9-10-2013

\title{
Detecting the Rapidly Expanding Outer Shell of the Crab Nebula: Where to Look
}

Xiang Wang

University of Kentucky, xiang.wang@uky.edu

Gary J. Ferland

University of Kentucky, gary@uky.edu

\section{J. A. Baldwin}

Michigan State University

E. D. Loh

Michigan State University

C. T. Richardson

Michigan State University

Follow this and additional works at: https://uknowledge.uky.edu/physastron_facpub

Part of the Astrophysics and Astronomy Commons, and the Physics Commons

Right click to open a feedback form in a new tab to let us know how this document benefits you.

\section{Repository Citation}

Wang, Xiang; Ferland, Gary J.; Baldwin, J. A.; Loh, E. D.; and Richardson, C. T., "Detecting the Rapidly Expanding Outer Shell of the Crab Nebula: Where to Look" (2013). Physics and Astronomy Faculty Publications. 58.

https://uknowledge.uky.edu/physastron_facpub/58

This Article is brought to you for free and open access by the Physics and Astronomy at UKnowledge. It has been accepted for inclusion in Physics and Astronomy Faculty Publications by an authorized administrator of UKnowledge. For more information, please contact UKnowledge@lsv.uky.edu. 
Detecting the Rapidly Expanding Outer Shell of the Crab Nebula: Where to Look

Digital Object Identifier (DOI)

https://doi.org/10.1088/0004-637X/774/2/112

Notes/Citation Information

Published in The Astrophysical Journal, v. 774, no. 2, 112, p. 1-13.

( $)$ 2013. The American Astronomical Society. All rights reserved. Printed in the U.S.A.

The copyright holder has granted permission for posting the article here.

This article is available at UKnowledge: https://uknowledge.uky.edu/physastron_facpub/58 


\title{
DETECTING THE RAPIDLY EXPANDING OUTER SHELL OF THE CRAB NEBULA: WHERE TO LOOK
}

\author{
Xiang Wang ${ }^{1}$, G. J. Ferland ${ }^{1}$, J. A. Baldwin ${ }^{2}$, E. D. LOH $^{2}$, and C. T. Richardson ${ }^{2}$ \\ ${ }^{1}$ Department of Physics and Astronomy, University of Kentucky, Lexington, KY 40506, USA; xiang.wang@uky.edu \\ ${ }^{2}$ Department of Physics and Astronomy, Michigan State University, East Lansing, MI 48824-2320, USA \\ Received 2013 March 22; accepted 2013 July 2; published 2013 August 21
}

\begin{abstract}
We present a range of steady-state photoionization simulations, corresponding to different assumed shell geometries and compositions, of the unseen postulated rapidly expanding outer shell to the Crab Nebula. The properties of the shell are constrained by the mass that must lie within it, and by limits to the intensities of hydrogen recombination lines. In all cases the photoionization models predict very strong emissions from high ionization lines that will not be emitted by the Crab's filaments, alleviating problems with detecting these lines in the presence of light scattered from brighter parts of the Crab. The near-NIR [Ne VI] $\lambda 7.652 \mu \mathrm{m}$ line is a particularly good case; it should be dramatically brighter than the optical lines commonly used in searches. The C IV $\lambda 1549$ doublet is predicted to be the strongest absorption line from the shell, which is in agreement with Hubble Space Telescope observations. We show that the cooling timescale for the outer shell is much longer than the age of the Crab, due to the low density. This means that the temperature of the shell will actually "remember" its initial conditions. However, the recombination time is much shorter than the age of the Crab, so the predicted level of ionization should approximate the real ionization. In any case, it is clear that IR observations present the best opportunity to detect the outer shell and so guide future models that will constrain early events in the original explosion.
\end{abstract}

Key words: ISM: supernova remnants - methods: numerical - supernovae: individual (SN1054)

Online-only material: color figure

\section{INTRODUCTION}

The Crab Nebula is generally thought to have been produced by a core collapse supernova. The total mass in the observed ejecta is $2-5 M_{\odot}$ (Davidson \& Fesen 1985; Fesen et al. 1997) and the pulsar should have a mass of about $1.4 M_{\odot}$ (Davidson \& Fesen 1985). This is much less than the total mass of 8-13 $M_{\odot}$ (Nomoto 1985, 1987; Kitaura et al. 2006) thought to be in the star before the explosion. Thus the long-standing problem: Where is the missing mass? The possibility most often discussed is that it lies within an unseen outer shell, sometimes referred to as the Crab's halo. The literature on this is comprehensive, with Lundqvist \& Tziamtzis (2012) and Smith (2013) giving good summaries of the current situation. Smith (2013) also discusses an alternative explanation, that the Crab was a type of under-luminous supernova.

There have only been a few predictions of the detailed spectrum of the outer shell. Lundqvist et al. (1986) did timedependent numerical simulations of the spectrum with a constant density structure and Sankrit \& Hester (1997) predicted some properties of a photoionized and shock-heated shell. Here we use an up-to-date atomic database in the spectral synthesis code Cloudy (Ferland et al. 2013) to compute emission and absorption spectra. We largely confirm previous estimates of the hydrogen emission but find that strong optical and infrared coronal lines should also be present. We identify promising lines in the IR that would be a robust indicator of the presence of this outer shell.

\section{PARAMETERS OF THE OUTER SHELL}

The total luminosity of the Crab Nebula, and its spectral energy distribution (SED), are well known (Davidson \& Fesen 1985). Although other energy sources such as shocks may be present (Sankrit \& Hester 1997), photoionization by this continuum must occur (the SED is observed) and by itself can power the outer shell. Shock heating would only add to this. To compute a photoionization model of the outer shell and its spectrum we must specify the gas composition, its density, and how the density varies with the radius.

We assume that the outer shell is an inhomogeneous shell with an uncertain outer radius, but with an inner radius equal to the outer radius of the familiar Crab, $R_{\text {in }}=5.0 \times 10^{18} \mathrm{~cm}$ (Sankrit \& Hester 1997). The expansion velocity at the inner radius $v_{\text {in }}$ is roughly $1680 \mathrm{~km} \mathrm{~s}^{-1}$ at this radius (the Crab is, of course, not a sphere, so this is a simplification).

A velocity gradient must be present, since the outer shell lies outside the familiar Crab. We consider both a Hubble flow, with $v(r) \propto r$, and an arbitrary velocity law as a sensitivity test, with $v(r) \propto r^{2}$. We obtain two different density laws from these two velocity distributions and apply them in this paper to check how predictions depend on this assumption.

The total mass in the outer shell may be of the order of $4-8 M_{\odot}$ (Sollerman et al. 2000). We assume $4 M_{\odot}$ recommended by Sollerman et al. (2000), which, as we show below, is consistent with limits to the line surface brightness (Fesen et al. 1997; Tziamtzis et al. 2009). We combine this with the three power laws given above to find the gas density as a function of the radius.

\subsection{The Outer Radius}

We will determine the gas density by combining the total mass with the density law and the inner and outer radii. The outer radius is unknown, but it must be specified to determine the gas density. Given our assumptions about the radius-velocity law, the outer radius will correspond to a particular highest expansion velocity. Chevalier (1977) gives a range of expansion velocities between $5000 \mathrm{~km} \mathrm{~s}^{-1}$ and $10,000 \mathrm{~km} \mathrm{~s}^{-1}$, Lundqvist et al. (1986) give a maximum expansion velocity of $5000 \mathrm{~km} \mathrm{~s}^{-1}$, Sankrit \& Hester (1997) assume a maximum velocity of $10,000 \mathrm{~km} \mathrm{~s}^{-1}$, 
Table 1

Basic Parameters of the Outer Shell for Three Difference Cases

\begin{tabular}{lccccc}
\hline \hline Case & $\gamma$ & $\alpha$ & $\beta$ & $\begin{array}{c}n_{0} \\
\left(\mathrm{~cm}^{-3}\right)\end{array}$ & \multicolumn{1}{c}{$\begin{array}{c}R_{\text {out }} \\
(\mathrm{cm})\end{array}$} \\
\hline I & 1 & -3 & 0 & 0.87 & $1.90 \mathrm{E}+19$ \\
II & 1 & -4 & 0 & 1.58 & $1.90 \mathrm{E}+19$ \\
III & 2 & -4 & -1 & 2.46 & $9.50 \mathrm{E}+18$
\end{tabular}

and Sollerman et al. (2000) quote $6370 \mathrm{~km} \mathrm{~s}^{-1}$. We assume that the velocity at the outer radius is $v_{\text {out }}=6370 \mathrm{~km} \mathrm{~s}^{-1}$, a velocity $\sim 3.8$ times larger than the expansion of the observed nebula, and give results relative to this velocity. The $v(r) \propto r$ Hubble flow results in

$$
R_{\text {out }} \approx 3.8 R_{\text {in }}=1.9 \times 10^{19}(\mathrm{~cm}),
$$

while for the $v(r) \propto r^{2}$ expansion law, the outer radius is

$$
R_{\text {out }} \approx 1.9 R_{\text {in }}=9.5 \times 10^{18}(\mathrm{~cm}) .
$$

\subsection{The Density Law}

For the spectroscopic simulations we need to set the outer shell density $n_{0}$ at its inner edge $R_{\text {in }}$, the density law $n(r) \propto r^{\alpha}$, and the outer radius $R_{\text {out }}$. We investigate two density laws here, $\alpha=-3$ and $\alpha=-4$. The density law is determined by two quantities: how the expansion velocity varies with radius, $v(r) \propto r^{\gamma}$, and how the mass flux varies with radius, MF $\propto r^{\beta}$. We consider three cases, summarized in Table 1, as follows.

1. The simplest case is a Hubble-law expansion, the sudden release of mass with a range of velocities so that $\gamma=1$ and $v \propto r$. For the mass flux, the simplest assumption is that the initial density distribution is constant, so that

$$
\mathrm{MF}=4 \pi r^{2} n(r) v(r) \propto 4 \pi r^{2+\alpha+\gamma}=4 \pi r^{\beta}
$$

is constant. Since $\gamma=1$, if $\alpha=-3$, then $\beta=0$, indicating mass flux conservation.

2. As the second case, we still assume that the Hubble velocity law is maintained so that $\gamma=1$ and $v \propto r$. If $\alpha=-4$, then $\beta=-1$, meaning that the mass flux decreases with increasing radius. This might happen if the outer layer of the star had a lower density.

3. As a third case we also consider $\alpha=-4$. Since we know that the expansion is accelerating (Trimble 1968), we will also investigate, as a sensitivity test, an arbitrarily different velocity law expansion with $\gamma=2$ and $v(r) \propto r^{2}$. In this case we also obtain $\beta=0$, that is, the mass flux is conserved.

The density law for case I is

$$
n(r)=n_{0}\left(\frac{r}{R_{\text {in }}}\right)^{\alpha}=n_{0}\left(\frac{r}{R_{\text {in }}}\right)^{-3}\left(\mathrm{~cm}^{-3}\right)
$$

and for cases II and III is

$$
n(r)=n_{0}\left(\frac{r}{R_{\text {in }}}\right)^{-4}\left(\mathrm{~cm}^{-3}\right) .
$$

\subsection{The Shell Mass and Inner Density}

We can calculate $n_{0}$ by mass conservation,

$$
M_{\text {halo }}=4 \pi \int_{R_{\text {in }}}^{R_{\text {out }}} m n_{0}\left(\frac{r}{R_{\text {in }}}\right)^{\alpha} r^{2} d r(\mathrm{gm}) .
$$

Here $M_{\text {halo }}$ is the total mass of the outer shell and $m$ is the mass per hydrogen for the assumed composition. Note that the composition of a supernova remnant is usually different in different parts; therefore we assume three different compositions for the outer shell: the abundances of some of the Crab filaments (Pequignot \& Dennefeld 1983), solar abundances (recommended by Sollerman et al. 2000), and interstellar medium (ISM) abundances (which are basically solar abundances with grains). If $\mu$ is the mass of the proton then $m=3.8 \mu$ for the enhanced Crab abundances derived by Pequignot $\&$ Dennefeld (1983) and $m=1.4 \mu$ for solar and ISM abundances. A list of assumed abundances is given in Table 3(a) in Pequignot \& Dennefeld (1983). We obtain the following expression for $n_{0}$ with the middle value $m=2.6 \mu$ and $M_{\text {halo }}=4 M_{\odot}$

$$
\begin{aligned}
& \left.\begin{array}{c}
n_{0}=\frac{M_{\text {halo }}}{4 \pi m R_{\text {in }}^{3} \ln \frac{R_{\text {out }}}{R_{\text {in }}}} \\
=0.87 \frac{2.6 \mu}{m} \frac{M_{\text {halo }}}{4 M_{\odot} \frac{\ln 3.8}{\ln \frac{R_{\text {out }}}{R_{\text {in }}}} \quad\left(\mathrm{cm}^{-3}\right)}
\end{array}\right\} \text { case I; } \\
& \left.\begin{array}{l}
n_{0}=\frac{M_{\text {halo }}}{4 \pi m R_{\text {in }}^{3}\left(1-\frac{R_{\text {in }}}{R_{\text {out }}}\right)} \\
58 \frac{2.6 \mu}{m} \frac{M_{\text {halo }}}{4 M_{\odot} \frac{0.74}{1-\frac{R_{\text {in }}}{R_{\text {out }}}} \quad\left(\mathrm{cm}^{-3}\right)}
\end{array}\right\} \text { case II; } \\
& n_{0}=\frac{M_{\text {halo }}}{4 \pi m R_{\text {in }}^{3}\left(1-\frac{R_{\text {in }}}{R_{\text {out }}}\right)} \\
& =2.46 \frac{2.6 \mu}{m} \frac{M_{\text {halo }}}{4 M_{\odot}} \frac{0.47}{1-\frac{R_{\text {in }}}{R_{\text {out }}}} \\
& \left.\left(\mathrm{cm}^{-3}\right)\right\} \\
& \text { case III }
\end{aligned}
$$

We see that the density depends on both the inner radius and the outer radius for the $\alpha=-3$ law. This is important because the density determines the emission measure of the lines, and this depends on the uncertain outer radius. For the case of $\alpha=-4$, the density depends only on the inner radius if the outer radius is much larger than the inner radius. Table 5 in Sollerman et al. (2000) also gave the densities in the inner edge for different density laws.

\subsection{Kinetic Energy}

Before proceeding with the model we derived the kinetic energy for each of these hypotheses. The kinetic energy of the filaments is about $3 \times 10^{49} \mathrm{erg}$ (Hester 2008), which is far less than the canonical $10^{51} \mathrm{erg}$ seen in the ejecta of core collapse supernovae (Davidson \& Fesen 1985). We calculate the kinetic energy in the outer shell to check if this makes up the missing energy. We obtain the kinetic energy of the outer shell using

$$
\begin{aligned}
& E_{k}=6.86 \times 10^{50} \frac{M_{\text {halo }}}{4 M_{\odot}} \frac{\ln 3.8}{\ln \frac{R_{\text {out }}}{R_{\text {in }}}} \frac{\left(\frac{R_{\text {out }}}{R_{\text {in }}}\right)^{2}-1}{13.44}(\mathrm{erg}), \text { case I; } \quad(10) \\
& E_{k}=4.26 \times 10^{50} \frac{M_{\text {halo }}}{4 M_{\odot}} \frac{0.74}{1-\frac{R_{\text {in }}}{R_{\text {out }}}} \frac{\frac{R_{\text {out }}}{R_{\text {in }}}-1}{2.8}(\mathrm{erg}), \text { case II; } \quad(11) \\
& E_{k}=6.70 \times 10^{50} \frac{M_{\text {halo }}}{4 M_{\odot}} \frac{0.47}{1-\frac{R_{\text {in }}}{R_{\text {out }}}} \frac{\left(\frac{R_{\text {out }}}{R_{\text {in }}}\right)^{3}-1}{6.38}(\mathrm{erg}), \text { case III. }
\end{aligned}
$$


These provide about half of the missing energy, which is within the uncertainty in our assumed shell parameters. Table 5 in Sollerman et al. (2000) also gave the kinetic energies of the outer shell for different density laws.

The next step is to predict the full emission and absorption line spectra of the outer shell using photoionization models.

\subsection{Emission Measure and Line Luminosity}

We obtain the luminosities of emission lines from the numerical calculations presented below. We use $\mathrm{H}$ I line emissivities given by Osterbrock \& Ferland (2006, hereafter AGN3) and Ferland (1980). The luminosity of $\mathrm{H} \beta$ is

$$
\begin{aligned}
& L(\mathrm{H} \beta)=\int \frac{4 \pi j_{\mathrm{H} \beta}}{n_{e} n_{p}} n(r)^{2} d V \\
& \approx \frac{4 \pi j_{\mathrm{H} \beta}}{n_{e} n_{p}} \times \mathrm{EM}\left(\mathrm{erg} \mathrm{s}^{-1}\right),
\end{aligned}
$$

where $4 \pi j_{\mathrm{H} \beta} / n_{e} n_{p}$ is the $\mathrm{H}$ I Case $\mathrm{B}$ recombination coefficient (AGN3). EM is the volume emission measure, defined as

$$
\begin{gathered}
\mathrm{EM}=\int n(r)^{2} d V \\
\approx \int\left[n_{0}\left(\frac{r}{R_{\mathrm{in}}}\right)^{\alpha}\right]^{2} d V\left(\mathrm{~cm}^{-3}\right)
\end{gathered}
$$

corresponding to

$$
\begin{aligned}
& \mathrm{EM}=\frac{4}{3} \pi n_{0}^{2} R_{\text {in }}^{3}\left[1-\left(\frac{R_{\text {in }}}{R_{\text {out }}}\right)^{3}\right] \\
& =3.89 \times 10^{56}\left(\frac{2.6 \mu}{m}\right)^{2}\left(\frac{M_{\text {halo }}}{4 M_{\odot}}\right)^{2} \quad \text { case I; } \\
& \times\left(\frac{\ln 3.8}{\ln \frac{R_{\text {out }}}{R_{\text {in }}}}\right)^{2} \frac{\left[1-\left(\frac{R_{\text {in }}}{R_{\text {out }}}\right)^{3}\right]}{0.98} \quad\left(\mathrm{~cm}^{-3}\right) \\
& \mathrm{EM}=\frac{4}{5} \pi n_{0}^{2} R_{\text {in }}^{3}\left[1-\left(\frac{R_{\text {in }}}{R_{\text {out }}}\right)^{5}\right] \\
& =7.86 \times 10^{56}\left(\frac{2.6 \mu}{m}\right)^{2}\left(\frac{M_{\text {halo }}}{4 M_{\odot}}\right)^{2} \quad \text { case II; } \\
& \times\left(\frac{0.74}{1-\frac{R_{\text {in }}}{R_{\text {out }}}}\right)^{2} \frac{\left[1-\left(\frac{R_{\text {in }}}{R_{\text {out }}}\right)^{5}\right]}{0.99} \quad\left(\mathrm{~cm}^{-3}\right) \\
& \mathrm{EM}=\frac{4}{5} \pi n_{0}^{2} R_{\text {in }}^{3}\left[1-\left(\frac{R_{\text {in }}}{R_{\text {out }}}\right)^{5}\right] \\
& \left.=1.82 \times 10^{57}\left(\frac{2.6 \mu}{m}\right)^{2}\left(\frac{M_{\text {halo }}}{4 M_{\odot}}\right)^{2}\right\} \text { case III. } \\
& \times\left(\frac{0.47}{1-\frac{R_{\text {in }}}{R_{\text {out }}}}\right)^{2} \frac{\left[1-\left(\frac{R_{\text {in }}}{R_{\text {out }}}\right)^{5}\right]}{0.96} \quad\left(\mathrm{~cm}^{-3}\right)
\end{aligned}
$$

Therefore we find the final expressions of the luminosity for $\mathrm{H} \beta$

$$
\begin{aligned}
L(\mathrm{H} \beta)= & 1.43 \times 10^{31}\left(\frac{T}{2.9 \times 10^{4}}\right)^{-1.20}\left(\frac{2.6 \mu}{m}\right)^{2}\left(\frac{M_{\text {halo }}}{4 M_{\odot}}\right)^{2} \\
& \times\left(\frac{\ln 3.8}{\ln \frac{R_{\text {out }}}{R_{\text {in }}}}\right)^{2} \frac{\left[1-\left(\frac{R_{\text {in }}}{R_{\text {out }}}\right)^{3}\right]}{0.98}\left(\mathrm{erg} \mathrm{s}^{-1}\right), \text { case I; (20) }
\end{aligned}
$$

$$
\begin{aligned}
L(\mathrm{H} \beta)= & 4.55 \times 10^{31}\left(\frac{T}{2.3 \times 10^{4}}\right)^{-0.833} \\
& \times\left(\frac{2.6 \mu}{m}\right)^{2}\left(\frac{M_{\text {halo }}}{4 M_{\odot}}\right)^{2}\left(\frac{0.74}{1-\frac{R_{\text {in }}}{R_{\text {out }}}}\right)^{2} \\
& \times \frac{\left[1-\left(\frac{R_{\text {in }}}{R_{\text {out }}}\right)^{5}\right]}{0.99}\left(\mathrm{erg} \mathrm{s}^{-1}\right), \text { case II; }
\end{aligned}
$$

$$
\begin{aligned}
L(\mathrm{H} \beta)= & 1.20 \times 10^{32}\left(\frac{T}{2 \times 10^{4}}\right)^{-0.833} \\
& \times\left(\frac{2.6 \mu}{m}\right)^{2}\left(\frac{M_{\text {halo }}}{4 M_{\odot}}\right)^{2}\left(\frac{0.47}{1-\frac{R_{\text {in }}}{R_{\text {out }}}}\right)^{2} \\
& \times \frac{\left[1-\left(\frac{R_{\text {in }}}{R_{\text {out }}}\right)^{5}\right]}{0.96}\left(\mathrm{erg} \mathrm{s}^{-1}\right), \text { case III; }
\end{aligned}
$$

where we suppose the temperature to be in the neighborhood of $2.9 \times 10^{4} \mathrm{~K}$ for case I, $2.3 \times 10^{4} \mathrm{~K}$ for case II, and $2 \times 10^{4} \mathrm{~K}$ for case III as computed below, and use the temperature powerlaw fit to $\left(4 \pi j_{\mathrm{H} \beta}\right) /\left(n_{e} n_{p}\right)$ given by Ferland (1980). This is approximate due to the assumption of Case B H I emission. We show below that the Lyman lines are not optically thick and that continuum fluorescent excitation is important.

\subsection{Scale Radius}

We can convert emission-line luminosities into surface brightness by dividing the luminosity by the area of emission on the sky. We assume that the lines form over a scale height determined by an effective radius, $R_{\text {eff }}$. The effective radius is defined as the position where half of the total line luminosity is formed. Emission line luminosities are determined by the emission measure, $n^{2} V$ (AGN3), so the inner highest-density regions are most important. We obtain the effective or "half luminosity" radius from

$$
\int_{R_{\mathrm{in}}}^{R_{\mathrm{eff}}} \frac{4 \pi j_{\mathrm{H} \beta}}{n_{e} n_{p}} n(r)^{2} d V=L / 2\left(\mathrm{erg} \mathrm{s}^{-1}\right) .
$$

If we move $\left(4 \pi j_{\mathrm{H} \beta}\right) /\left(n_{e} n_{p}\right)$ out of the integral, equivalent to assuming that the temperature is constant, we find

$$
\left.\begin{array}{c}
R_{\text {eff }}=R_{\text {in }}\left(\frac{2}{1+\left(\frac{R_{\text {in }}}{R_{\text {out }}}\right)^{3}}\right)^{1 / 3} \\
=6.26 \times 10^{18} \frac{\left(\frac{2}{1+\left(\frac{R_{\text {in }}}{R_{\text {out }}}\right)^{3}}\right)^{1 / 3}}{1.25}(\mathrm{~cm}) \\
=1.25 R_{\text {in }}
\end{array}\right\} \text { cases I and II; }
$$




\subsection{Average Surface Brightness in H I Recombination Lines}

We convert the luminosities given above into surface brightness averaged over the full outer shell as it would be seen projected on the sky, in order to compare the results with observations. We obtain the surface brightness

$$
S(\mathrm{H} \beta)=\frac{1}{k^{2}} \frac{L}{4 \pi^{2} R_{e f f}^{2}}\left(\operatorname{erg~s}^{-1} \mathrm{~cm}^{-2} \operatorname{arcsec}^{-2}\right)
$$

corresponding to

$$
\begin{aligned}
& S(\mathrm{H} \beta)=2.76 \times 10^{-19}\left(\frac{T}{2.9 \times 10^{4}}\right)^{-1.20}\left(\frac{2.6 \mu}{m}\right)^{2}\left(\frac{M_{\text {halo }}}{4 M_{\odot}}\right)^{2} \\
& \times\left(\frac{\ln 3.8}{\ln \frac{R_{\text {out }}}{R_{\text {in }}}}\right)^{2} \frac{\left[1-\left(\frac{R_{\text {in }}}{R_{\text {out }}}\right)^{3}\right]}{0.98} \frac{1.25}{\left[\frac{2}{1+\left(\frac{R_{\text {in }}}{R_{\text {out }}}\right)^{3}}\right]^{1 / 3}} \\
& \times\left(\operatorname{erg~s}^{-1} \mathrm{~cm}^{-2} \operatorname{arcsec}^{-2}\right), \text { case I; } \\
& S(\mathrm{H} \beta)=9.42 \times 10^{-19}\left(\frac{T}{2.3 \times 10^{4}}\right)^{-0.833}\left(\frac{2.6 \mu}{m}\right)^{2} \\
& \times\left(\frac{M_{\text {halo }}}{4 M_{\odot}}\right)^{2}\left(\frac{0.74}{1-\frac{R_{\text {in }}}{R_{\text {out }}}}\right)^{2} \frac{\left[1-\left(\frac{R_{\text {in }}}{R_{\text {out }}}\right)^{5}\right]}{0.99} \\
& \times \frac{1.15}{\left[\frac{2}{1+\left(\frac{R_{\text {in }}}{R_{\text {out }}}\right)^{5}}\right]^{1 / 5}}\left(\operatorname{erg~s}^{-1} \mathrm{~cm}^{-2} \operatorname{arcsec}^{-2}\right) \text {, case II; }
\end{aligned}
$$

and

$$
\begin{aligned}
S(\mathrm{H} \beta)= & 2.17 \times 10^{-18}\left(\frac{T}{2 \times 10^{4}}\right)^{-0.833}\left(\frac{2.6 \mu}{m}\right)^{2}\left(\frac{M_{\text {halo }}}{4 M_{\odot}}\right)^{2} \\
& \times\left(\frac{0.47}{1-\frac{R_{\text {in }}}{R_{\text {out }}}}\right)^{2} \frac{\left[1-\left(\frac{R_{\text {in }}}{R_{\text {out }}}\right)^{5}\right]}{0.96} \frac{1.15}{\left[\frac{2}{1+\left(\frac{R_{\text {in }}}{R_{\text {out }}}\right)^{5}}\right]^{1 / 5}}
\end{aligned}
$$$$
\left(\operatorname{erg~s}^{-1} \mathrm{~cm}^{-2} \operatorname{arcsec}^{-2}\right) \text {, case III; }
$$

where $k=206,265$ converts luminosity into surface brightness. Table 5 in Sollerman et al. (2000) and Tziamtzis et al. (2009) also gave the surface brightness of the outer shell for different density laws.

The upper limit to the $\mathrm{H} \beta$ surface brightness corresponds to an upper limit to the mass in the shell for a given power-law index. The composition also affects $S(\mathrm{H} \beta)$ because, for Crab abundances, the heavy elements contribute to the total mass. This means that the hydrogen density and $S(\mathrm{H} \beta)$ are lower for the same mass but higher $Z$. The $\mathrm{H} \beta$ surface brightness is highest for models with solar abundances, where more of the $4 M_{\odot}$ is $\mathrm{H}$ so the density is higher. The coefficients in Equations (27)-(29) were evaluated for abundances intermediate between solar and Crab. The maximum expansion velocity also affects the surface brightness because it sets the outer radius that appears in the equations. A shell with a larger expansion velocity is more spread out and has lower density and lower $S(\mathrm{H} \beta)$.

With these assumptions the physical conditions in the outer shell, the ionization, and the temperature can be computed. The observations described below suggest that the upper limit to $\mathrm{H} \beta$ is about $S(\mathrm{H} \beta)<4 \times 10^{-18} \mathrm{erg} \mathrm{cm}^{-2} \mathrm{~s}^{-1} \operatorname{arcsec}^{-2}$. If we apply different values of $m$, indicating different abundances, into Equations (27)-(29), we find that cases I and II have average surface brightness that are less than this observed limit for all abundances. Case III has a surface brightness that is under this observed limit for Crab abundances but above this observed limit for solar and ISM abundances. We consider all these models in the following to examine their predictions.

\section{MODEL CALCULATIONS}

Here we will consider models with various compositions and power laws to compute the emitted spectrum. Cases I and II are more consistent with the existence of a large mass, $4 M_{\odot}$, and the limits to the surface brightness (Fesen et al. 1997; Tziamtzis et al. 2009). Since they have similar results for all kinds of calculations, we only give the full results for case I as an example. Sollerman et al. (2000) say that solar abundances might be most appropriate if the outer shell comes from the upper envelope of the star. We adopt this and further assume that grains have not formed in the fast wind. We present results for all the scenarios below, but will focus on this single model.

\subsection{The Emission-line Spectrum}

We use version 13 of the plasma simulation code Cloudy (Ferland et al. 2013) to predict the observed spectrum. We compute the luminosities of many emission lines and convert them to surface brightness by dividing the luminosities by the size derived above. We obtain different emission lines and surface brightness for the three different models. Figure 1 shows predicted spectra integrated over the full outer shell for case I with solar abundances. The upper panel shows the full range $0.1-100 \mu \mathrm{m}$. The lower panel shows the range $1-30 \mu \mathrm{m}$ in greater detail. We focus on the UV-IR spectral region because this would be easiest to study with today's instrumentation.

Tables 2-10 give the average surface brightnesses $S$ for IR, optical, and UV emission lines, as defined by Equations (27)-(29), for models with different abundances. These can be compared to the best observational upper limit achieved to date for the outer shell, $S<1.2 \times 10^{-17} \mathrm{erg} \mathrm{cm}^{-2} \mathrm{~s}^{-1} \operatorname{arcsec}^{-2}$ using longslit spectra to search for $\mathrm{H} \alpha$ (Fesen et al. 1997, but with their value adjusted upward by a factor of 3.4 to correct for the observed extinction; Tziamtzis et al. 2009). All lines at or brighter than that limit are italicized in Tables 2-10.

$\mathrm{H} \alpha$ is brighter than the observational limit in case III with solar or ISM abundances, so at face value these models appear to be ruled out, at least for an outer shell containing the full amount of the missing mass. However, scattered light from the much brighter parts of the Crab is a major issue, as has been discussed by Tziamtzis et al. (2009). The Fesen et al. (1997) upper limit really corresponds to radii beyond about 0.3 from the bright edge of the main nebula $\left(R_{\text {in }}\right)$, because their spectrum inside that radius is likely to be dominated by an unknown amount of scattered light. Figure 2 shows an example of the emissivity in three emission lines as a function of the depth into the shell from its inner edge at $R_{\text {in }}$ for case I with solar abundances. Figure 3 shows the results of integrating these emissivities along the line of sight through the outer shell to 

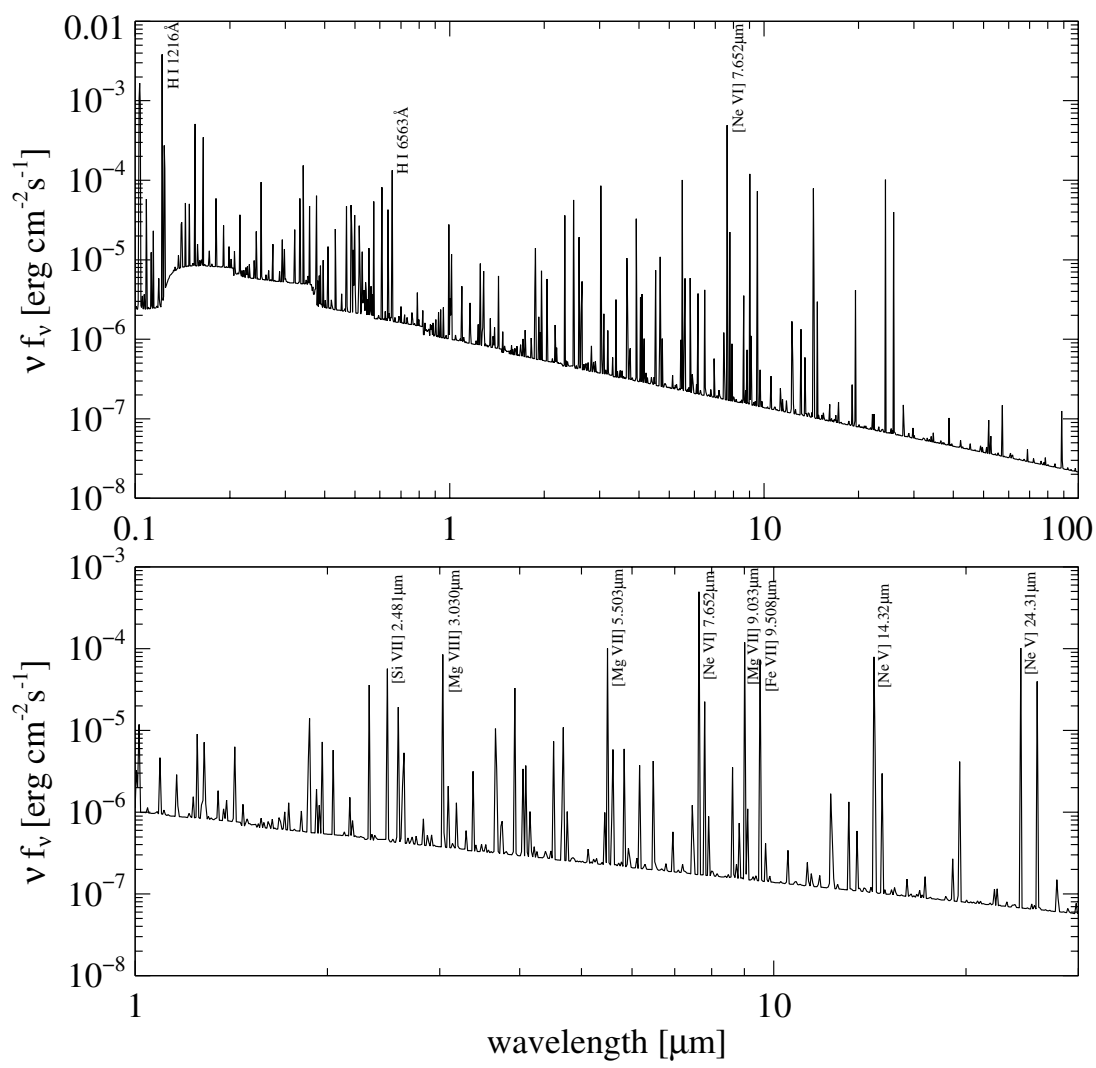

Figure 1. The upper panel shows emission lines from Crab outer shell between the wavelength of $0.1 \mu \mathrm{m}$ and $100 \mu \mathrm{m}$ for case I with solar abundances. HI $\lambda 1216$, $\mathrm{HI} \lambda 6563$, and [Ne VI] $7.652 \mu \mathrm{m}$ are the strongest lines in UV, optical, and IR bands respectively. The lower panel shows the emission lines for the same model in the range between $1 \mu \mathrm{m}$ and $30 \mu \mathrm{m}$ and all the lines that are brighter than the $\mathrm{H} \beta$ line are marked on the figure.

Table 2

Predicted IR Emission Line Average Surface Brightness, Sorted by Surface Brightness for Each Model, for All Lines Brighter than H $\beta$, for Case I

\begin{tabular}{|c|c|c|c|c|c|}
\hline \multicolumn{2}{|c|}{ Crab Abund. } & \multicolumn{2}{|c|}{ Solar Abund. } & \multicolumn{2}{|c|}{ ISM Abund. } \\
\hline $\begin{array}{l}\text { Line }^{\mathrm{a}} \\
{[\mathrm{Ne} \text { VI] } 7.652 m}\end{array}$ & $\begin{array}{c}\text { Surf. Br. }^{b} \\
1 E-17\end{array}$ & $\begin{array}{c}\text { Line } \\
{[\mathrm{Ne} \text { VI }] 7.652 \mathrm{~m}}\end{array}$ & $\begin{array}{c}\text { Surf. Br. } \\
1 E-17\end{array}$ & $\begin{array}{c}\text { Line } \\
{[\mathrm{Ne} \text { VI] } 7.652 \mathrm{~m}}\end{array}$ & $\begin{array}{c}\text { Surf. Br. } \\
1 E-17\end{array}$ \\
\hline$[\mathrm{Ne} \mathrm{v}] 24.31 \mathrm{~m}$ & $2 \mathrm{E}-18$ & {$[\mathrm{Mg}$ VII] $9.033 \mathrm{~m}$} & $3 \mathrm{E}-18$ & {$[\mathrm{Ne} \mathrm{v}] 24.31 \mathrm{~m}$} & $3 \mathrm{E}-18$ \\
\hline$[\mathrm{Mg}$ VIII $] 3.030 \mathrm{~m}$ & $2 E-18$ & {$[\mathrm{Ne} \mathrm{v}] 24.31 \mathrm{~m}$} & $3 E-18$ & {$[\mathrm{Ne} \mathrm{v}] 14.32 \mathrm{~m}$} & $2 \mathrm{E}-18$ \\
\hline [Mg VII] $9.033 \mathrm{~m}$ & $2 \mathrm{E}-18$ & {$[\mathrm{Mg}$ VII $] 5.503 \mathrm{~m}$} & $3 E-18$ & [S VIII] 9914 & $1 \mathrm{E}-18$ \\
\hline$[\mathrm{Mg}$ VII $] 5.503 \mathrm{~m}$ & $2 \mathrm{E}-18$ & {$[\mathrm{Mg}$ VIII $] 3.030 \mathrm{~m}$} & $2 \mathrm{E}-18$ & {$[\mathrm{Mg}$ VII $] 9.033 \mathrm{~m}$} & $1 E-18$ \\
\hline$[\mathrm{Ne} \mathrm{v}] 14.32 \mathrm{~m}$ & $2 \mathrm{E}-18$ & {$[\mathrm{Ne} \mathrm{v}] 14.32 \mathrm{~m}$} & $2 \mathrm{E}-18$ & & \\
\hline He II $1.012 \mathrm{~m}$ & $5 \mathrm{E}-19$ & {$[\mathrm{Fe}$ VII $] 9.508 \mathrm{~m}$} & $2 \mathrm{E}-18$ & & \\
\hline [O IV $] 25.88 \mathrm{~m}$ & $5 \mathrm{E}-19$ & [Si VII] $2.481 \mathrm{~m}$ & $1 \mathrm{E}-18$ & & \\
\hline [Fe VII] $9.508 \mathrm{~m}$ & $4 \mathrm{E}-19$ & & & & \\
\hline [Si IX] 3.929m & $4 \mathrm{E}-19$ & & & & \\
\hline [S VIII] 9914 & $3 \mathrm{E}-19$ & & & & \\
\hline [Si VII] $2.481 \mathrm{~m}$ & $3 \mathrm{E}-19$ & & & & \\
\hline
\end{tabular}

Notes.

Italicized entries have predicted surface brightness at or above the current optical-passband detection limit.

a Wavelengths are given in $\AA$ unless noted with $\mathrm{m}=$ microns

${ }^{\mathrm{b}}$ Surface brightness, erg cm ${ }^{-2} \mathrm{~s}^{-1} \operatorname{arcsec}^{-2}$.

find the predicted surface brightness as a function of $R_{\text {proj }} / R_{\text {in }}$ for cases I, II, and III. We used solar abundances and assumed a distance of $2 \mathrm{kpc}$ and a spherical shell. Note that Figure 3 is shown with linear scales for both radius and surface brightness, to make the wide range in surface brightness more obvious, and that each panel has been separately scaled in surface brightness. Each panel also shows the Fesen et al. (1997) H $\alpha$ upper limit as a horizontal line beginning at a point 0.3 beyond $R_{\text {in }}$. The lack of an $\mathrm{H} \alpha$ detection does nothing to rule out cases I or
II, nor does it firmly rule out case III. Further ground-based observations might be able to push these optical-passband limits slightly fainter, but observations in $\mathrm{H} \alpha$ or other lines that are also emitted by the main part of the nebula will require great attention to the scattered light issue.

What is needed are unique spectroscopic tracers in the form of high-ionization lines not emitted by the filaments or (hopefully) by the thin [O III]-emitting skin (Sankrit \& Hester 1997) that surrounds the outer edge of the synchrotron bubble. Our models 


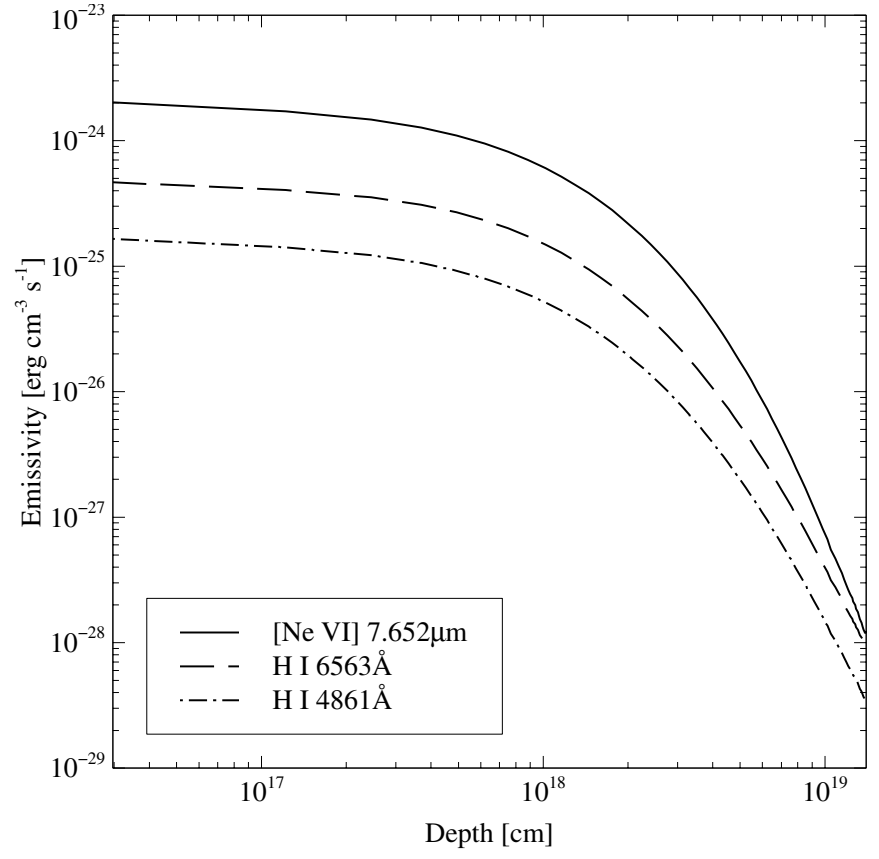

Figure 2. Emissivity as a function of depth of lines [Ne vI] $7.652 \mu \mathrm{m}$, H I $\lambda 6563$, and $\mathrm{HI}_{\mathrm{I}} \lambda 4861$, for case I with solar abundances. For a distance of $2 \mathrm{kpc}, 2 \times 10^{18} \mathrm{~cm}$ corresponds to 1 !' 1 .

Table 3

Predicted Optical Emission Line Average Surface Brightness, Sorted by Surface Brightness for Each Model, for All Lines Brighter than $\mathrm{H} \beta$, for Case I

\begin{tabular}{|c|c|c|c|c|c|}
\hline \multicolumn{2}{|c|}{ Crab Abund. } & \multicolumn{2}{|c|}{ Solar Abund. } & \multicolumn{2}{|c|}{ ISM Abund. } \\
\hline Line $^{a}$ & Surf. Br. ${ }^{b}$ & Line & Surf. Br. & Line & Surf. Br. \\
\hline He II 4686 & $2 \mathrm{E}-18$ & Н г 6563 & $3 \mathrm{E}-18$ & Н І 6563 & $3 \mathrm{E}-18$ \\
\hline Н г 6563 & $8 \mathrm{E}-19$ & Fe vII 6087 & $2 \mathrm{E}-18$ & Н I 4861 & $1 \mathrm{E}-18$ \\
\hline Fe $\times 6375$ & 7E-19 & Fe VII 5721 & $1 \mathrm{E}-18$ & & \\
\hline Fe VII 6087 & $5 \mathrm{E}-19$ & Н г 4861 & $1 \mathrm{E}-18$ & & \\
\hline Fe VII 5721 & $3 \mathrm{E}-19$ & & & & \\
\hline H I 4861 & $3 \mathrm{E}-19$ & & & & \\
\hline
\end{tabular}

Notes.

a Wavelengths are given in $\AA$ unless noted with $\mathrm{m}=$ microns.

b Surface brightness, erg cm ${ }^{-2} \mathrm{~s}^{-1} \operatorname{arcsec}^{-2}$.

predict strong emission lines from high-ionization species of $\mathrm{C}, \mathrm{N}, \mathrm{O}, \mathrm{Ne}, \mathrm{Si}, \mathrm{Mg}$, and $\mathrm{Fe}$, principally in the UV and IR parts of the spectrum. A surface brightness limit similar to that for $\mathrm{H} \alpha$ might be reachable in a few UV lines, notably C IV $\lambda \lambda 1548,1551$, in about $3 \mathrm{hr}$ of on-target exposure (plus an equal sky exposure) using Hubble Space Telescope/Advanced Camera for Surveys (HST/ACS) imaging with very heavy ondetector binning. But the most promising lines are in the mid-IR, particularly [Ne vI] $7.652 \mu \mathrm{m}$, which is also shown on Figure 3. Although these IR lines are somewhat fainter than the UV lines, they could be targeted with either SOFIA or (eventually) JWST mid-IR imagers and spectrographs. Archival Spitzer images and long-slit spectra also exist, and might be worth co-adding to search for these lines. Firm statements could be made about cases II or III if an IR measurement as deep as the $\mathrm{H} \alpha$ limit could be obtained.

An alternative to searching areas off to the side of the main part of the Crab would be to obtain spectra averaging over a fairly large area at the center of the Crab, where the projected

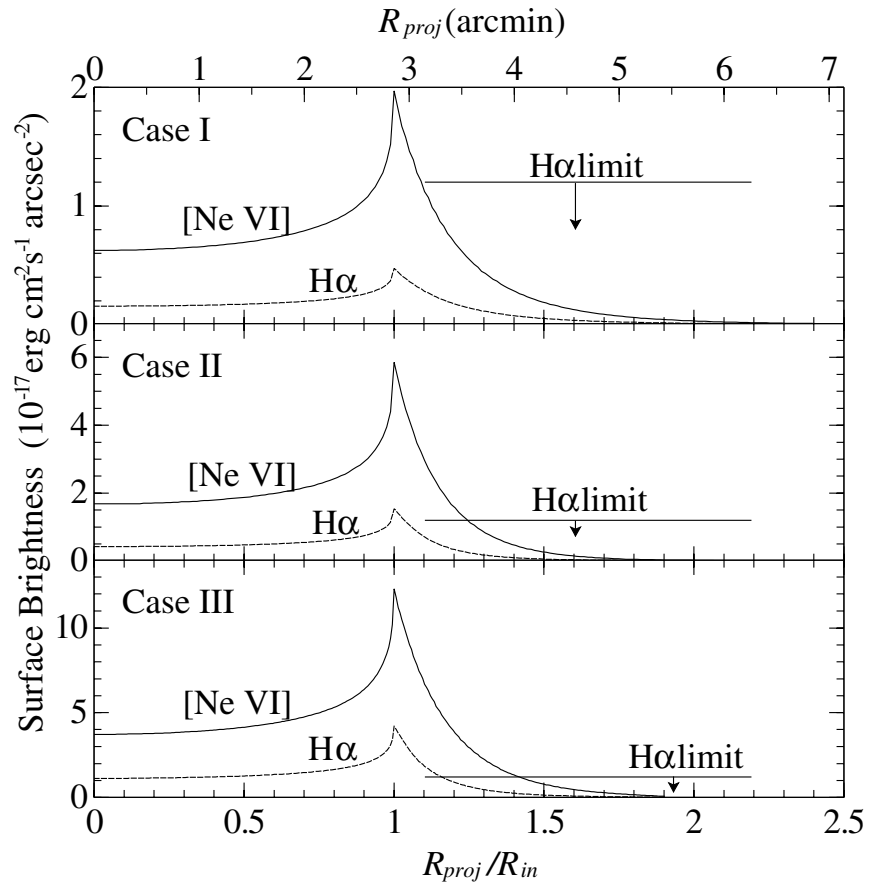

Figure 3. Predicted surface brightness of the [Ne vI] $7.652 \mu \mathrm{m}$ and $\mathrm{H} \alpha$ emission lines, as a function of $R_{\text {proj }}$, the radial distance from the center of expansion as seen projected on the sky. These are computed for cases I, II, and III with solar abundances, assuming a distance of $2 \mathrm{kpc}$ and a spherical outer shell with inner radius $R_{\text {in }}=5 \times 10^{18} \mathrm{~cm}$. The surface brightness for $R_{\text {proj }}<R_{\text {in }}$ includes both the front and rear sides of the outer shell. The horizontal bar in each panel shows the Fesen et al. (1997) $\mathrm{H} \alpha$ upper limit discussed in the text, starting at a point 0.3 beyond $R_{\text {in }}$ and extending to the end of the slit.

expansion velocities are toward and away from us, and searching for these mid-IR lines with positive and negative velocity shifts corresponding to the shell structure. Lundqvist \& Tziamtzis (2012) used this method in the optical passband to search for [O III] and Ca II lines. The [Ne VI] $7.652 \mu \mathrm{m}$ line falls within the spectral range covered by the Spitzer/IRS, but Temim et al. (2012) do not report any strong feature at this wavelength in their IRS spectra of the Crab. The predicted spectral signature for such emission lines would be two broad peaks displaced symmetrically around the Crab's heliocentric systemic velocity of about $0 \mathrm{~km} \mathrm{~s}^{-1}$ and separated by about $4000 \mathrm{~km} \mathrm{~s}^{-1}$. We are in the process of carrying out the very careful reanalysis of the Spitzer spectra needed to search for faint features of this type. However, the low velocity resolution $\left(4700 \mathrm{~km} \mathrm{~s}^{-1}\right)$ may prevent a clear distinction between any emission from an outer shell and emission from the ionized outer skin of the main part of the Crab (see Lundqvist \& Tziamtzis 2012, their Figure 9).

Cloudy predicts the intensity of $\mathrm{H}$ I lines including line optical depths effect, collisional excitation and de-excitation, and continuum fluorescent excitation. The predicted $\mathrm{H}_{\mathrm{I}}$ intensities can be compared with Case B (pure recombination in which Lyman lines are optically thick) and Case A (Lyman lines are optically thin and there is no continuum fluorescent excitation).

Table 11 compares $\mathrm{H}$ I luminosities for the solar abundance of the case I Crab shell. It gives the computed luminosities with all processes included, along with the luminosities obtained from the computed density and temperature and assuming Cases A and B emission (Storey \& Hummer 1995).The predicted lines are about $10 \%-140 \%$ brighter than Case B, an indication that continuum fluorescent excitation is important. The Lyman lines 
Table 4

Predicted UV Emission Line Average Surface Brightness, Sorted by Surface Brightness for Each Model, for All Lines Brighter than H $\beta$, for Case I

\begin{tabular}{|c|c|c|c|c|c|}
\hline \multicolumn{2}{|c|}{ Crab Abund. } & \multicolumn{2}{|c|}{ Solar Abund. } & \multicolumn{2}{|c|}{ ISM Abund. } \\
\hline Line $^{a}$ & Surf. Br. ${ }^{b}$ & Line & Surf. Br. & Line & Surf. Br. \\
\hline$O$ VI $1032+1038$ & $1 E-16$ & $O$ VI $1032+1038$ & $2 E-16$ & $H_{I} 1216$ & $9 E-17$ \\
\hline CIV $1548+1551$ & $1 E-16$ & H I 1216 & $9 E-17$ & $O$ VI $1032+1038$ & $9 E-17$ \\
\hline HI 1216 & $4 E-17$ & HI 1026 & $3 E-17$ & CIV $1548+1551$ & $3 E-17$ \\
\hline He II 1640 & $1 E-17$ & CIV $1548+1551$ & $2 E-17$ & H I 1026 & $3 E-17$ \\
\hline$O \vee 1211+1218$ & $1 E-17$ & $N \vee 1239+1243$ & $2 E-17$ & $N \vee 1239+1243$ & $2 E-17$ \\
\hline$N \vee 1239+1243$ & $1 E-17$ & $O \vee 1211+1218$ & $1 E-17$ & $O v 1211+1218$ & $1 E-17$ \\
\hline Hi 1026 & $9 \mathrm{E}-18$ & He II 1640 & $9 \mathrm{E}-18$ & He II 1640 & $8 \mathrm{E}-18$ \\
\hline He II 1215 & $5 \mathrm{E}-18$ & {$[\mathrm{Ne} \mathrm{v}] 3426$} & $3 \mathrm{E}-18$ & {$[\mathrm{Ne} \mathrm{v}] 3426$} & $4 \mathrm{E}-18$ \\
\hline$[\mathrm{Ne} \mathrm{v}] 3426$ & $3 E-18$ & He II 1215 & $3 \mathrm{E}-18$ & He II 1215 & $3 \mathrm{E}-18$ \\
\hline He II 1085 & $2 \mathrm{E}-18$ & Mg VII 2569 & $2 \mathrm{E}-18$ & Ne v 3346 & $2 \mathrm{E}-18$ \\
\hline $\mathrm{C}_{\text {III] }} 1907+1910$ & $2 \mathrm{E}-18$ & Fe VII 3759 & $2 \mathrm{E}-18$ & He II 1085 & $1 \mathrm{E}-18$ \\
\hline Mg VII 2569 & $2 \mathrm{E}-18$ & He II 1085 & $1 \mathrm{E}-18$ & & \\
\hline He II 1025 & $1 \mathrm{E}-18$ & Ne v 3346 & $1 \mathrm{E}-18$ & & \\
\hline Ne v 3346 & $1 E-18$ & [Mg VI] 1806 & $1 \mathrm{E}-18$ & & \\
\hline C v 1312 & $1 E-18$ & & & & \\
\hline He II 2050 & $1 \mathrm{E}-18$ & & & & \\
\hline$[\mathrm{C} \mathrm{v}] 2271+2275$ & $8 E-19$ & & & & \\
\hline He II 3203 & $8 \mathrm{E}-19$ & & & & \\
\hline Ne v 1141 & $7 E-19$ & & & & \\
\hline$[\mathrm{Mg} \mathrm{VI}] 1806$ & $7 E-19$ & & & & \\
\hline Si VIII 1446 & $5 E-19$ & & & & \\
\hline He II 2733 & $4 \mathrm{E}-19$ & & & & \\
\hline C VI 1240 & $4 \mathrm{E}-19$ & & & & \\
\hline Fe VII 3759 & $4 \mathrm{E}-19$ & & & & \\
\hline He II 3645 & $3 \mathrm{E}-19$ & & & & \\
\hline O IV 1405 & $3 E-19$ & & & & \\
\hline [Fe VII] 3586 & $3 E-19$ & & & & \\
\hline
\end{tabular}

Notes. Italicized entries have predicted surface brightness at or above the current optical-passband detection limit.

a Wavelengths are given in $\AA$ unless noted with $\mathrm{m}=$ microns.

b Surface brightness, erg cm ${ }^{-2} \mathrm{~s}^{-1} \operatorname{arcsec}^{-2}$.

Table 5

Predicted IR Emission Line Average Surface Brightness, Sorted by Surface Brightness for Each Model, for All Lines Brighter than H $\beta$, for Case II

\begin{tabular}{|c|c|c|c|c|c|}
\hline \multicolumn{2}{|c|}{ Crab Abund. } & \multicolumn{2}{|c|}{ Solar Abund. } & \multicolumn{2}{|c|}{ ISM Abund. } \\
\hline$[\mathrm{Ne}$ VI] $7.652 \mathrm{~m}$ & $5 E-17$ & {$[\mathrm{Ne}$ VI] $7.652 \mathrm{~m}$} & $4 E-17$ & {$[\mathrm{Ne}$ VI] $7.652 \mathrm{~m}$} & $4 E-17$ \\
\hline$[N e \mathrm{v}] 24.31 \mathrm{~m}$ & $1 E-17$ & {$[\mathrm{Ne} \mathrm{v}] 24.31 \mathrm{~m}$} & $1 E-17$ & {$[\mathrm{Ne} v] 24.31 \mathrm{~m}$} & $2 E-17$ \\
\hline$[\mathrm{Ne} v] 14.32 \mathrm{~m}$ & $1 E-17$ & {$[\mathrm{Ne} v] 14.32 \mathrm{~m}$} & $1 E-17$ & {$[\mathrm{Ne}$ v] $14.32 \mathrm{~m}$} & $1 E-17$ \\
\hline [O IV] $25.88 \mathrm{~m}$ & $5 E-18$ & [O IV] $25.88 \mathrm{~m}$ & $9 \mathrm{E}-18$ & [O IV] $25.88 \mathrm{~m}$ & $5 E-18$ \\
\hline$[\mathrm{Mg}$ VII $] 5.503 \mathrm{~m}$ & $4 \mathrm{E}-18$ & [Mg VII $] 9.033 \mathrm{~m}$ & $6 \mathrm{E}-18$ & & \\
\hline [Mg VIII] $3.030 \mathrm{~m}$ & $3 E-18$ & {$[\mathrm{Mg} \mathrm{VII}] 5.503 \mathrm{~m}$} & $5 E-18$ & & \\
\hline [Fe VII] $9.508 \mathrm{~m}$ & $2 \mathrm{E}-18$ & [Si VII] $2.481 \mathrm{~m}$ & $4 \mathrm{E}-18$ & & \\
\hline He II $1.012 \mathrm{~m}$ & $1 \mathrm{E}-18$ & & & & \\
\hline [Si VII $] 2.481 \mathrm{~m}$ & $1 E-18$ & & & & \\
\hline
\end{tabular}

Notes. Italicized entries have predicted surface brightness at or above the current optical-passband detection limit.

a Wavelengths are given in $\AA$ unless noted with $\mathrm{m}=$ microns.

b Surface brightness, erg cm ${ }^{-2} \mathrm{~s}^{-1} \operatorname{arcsec}^{-2}$.

in the outer shell are not optically thick so continuum pumping is important, causing them to be brighter than would be found with pure recombination. The optical depth in $\mathrm{L} y \beta$, for instance, is about 1 , so neither Case A nor Case B formally apply. The predicted deviations are not large and Case B is, as is often the case, a fair approximation to the actual emission.

\subsection{Gas Temperature}

Figure 4 shows the gas kinetic temperature across the outer shell. It increases as the depth increases for all three models. This is because the Crab radiation field, which powers the outer shell, decreases at $r^{-2}$, because of the inverse square law. The gas density falls off faster, as $r^{-3}$ or $r^{-4}$. As a result the ionization 
Table 6

Predicted Optical Emission Line Average Surface Brightness, Sorted by Surface Brightness for Each Model, for All Lines Brighter than $\mathrm{H} \beta$, for Case II.

\begin{tabular}{|c|c|c|c|c|c|}
\hline \multicolumn{2}{|c|}{ Crab Abund. } & \multicolumn{2}{|c|}{ Solar Abund. } & \multicolumn{2}{|c|}{ ISM Abund. } \\
\hline Line $^{a}$ & Surf. Br. ${ }^{b}$ & Line & Surf. Br. & Line & Surf. Br. \\
\hline He II 4686 & $6 \mathrm{E}-18$ & H I 6563 & $9 \mathrm{E}-18$ & H I 6563 & $9 E-18$ \\
\hline Н г 6563 & $2 \mathrm{E}-18$ & Fe VII 6087 & $7 E-18$ & Н г 4861 & $3 E-18$ \\
\hline Fe VII 6087 & $2 \mathrm{E}-18$ & Fe VII 5721 & $4 \mathrm{E}-18$ & & \\
\hline Fe viI 5721 & $1 E-18$ & Н г 4861 & $3 E-18$ & & \\
\hline Н г 4861 & $8 \mathrm{E}-19$ & & & & \\
\hline
\end{tabular}

Notes.

a Wavelengths are given in $\AA$ unless noted with $\mathrm{m}=$ microns.

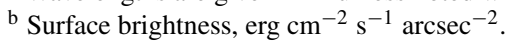

parameter, the ratio of photon to hydrogen densities (AGN3), increases as $r$ increases. Higher ionization parameter gas tends to be hotter.

\subsection{The Absorption Line Spectrum}

We compute optical depths for different assumptions about the expansion velocities. Tables 12 to 14 give the optical depths for models with Crab abundances, solar abundances, and ISM abundances, respectively. We continue to focus on the models with solar abundances. From Tables 12 to 14 we see that the optical depths for the $\mathrm{C}$ IV $\lambda \lambda 1549$ doublet are not much greater than 1 . The lines mainly form over a small radius due to the density decline, so the wind acceleration should not be large over the line forming region. We make two assumptions to estimate the optical depth. First we assume a static shell, in which the lines are only thermally broadened. This would apply if there was no acceleration across the layer where the lines form. In this case there is sufficient opacity to produce the observed lines. In particular, the C IV $\lambda \lambda 1549$ doublet has an optical depth of 2.68, consistent with the Sollerman et al. (2000) tentative detection. We note that the optical depth of the O VI $\lambda \lambda 1034$ doublet is much larger than 1 , which indicates strong absorption at that wavelength.

If the lines have a significant component of turbulence or if the expansion velocity changes across the line-forming region then the lines will be spread over a wider velocity range. Here the lines are optically thin.

Table 15 gives the computed line optical depths for both static and dynamic cases. We find the optical depth to be very small if we add a turbulence with velocity $v=1680 \mathrm{~km} \mathrm{~s}^{-1}$ as the expansion velocity of the inner radius of the outer shell. The O VI $\lambda \lambda 1034$ doublet then becomes optically thin as well.

The truth will lie between these two limiting assumptions. We will consider dynamic models, in which the velocity is determined self consistently, in future papers.

\subsection{Is Steady State Appropriate?}

\subsubsection{Recombination Timescale}

The recombination timescale is defined as (AGN3)

$$
t_{\mathrm{rec}}=\frac{1}{n_{e} \alpha_{\mathrm{B}}\left(T_{e}\right)}
$$

Table 7

Predicted UV Emission Line Average Surface Brightness, Sorted by Surface Brightness for Each Model, for All Lines Brighter than H $\beta$, for Case II

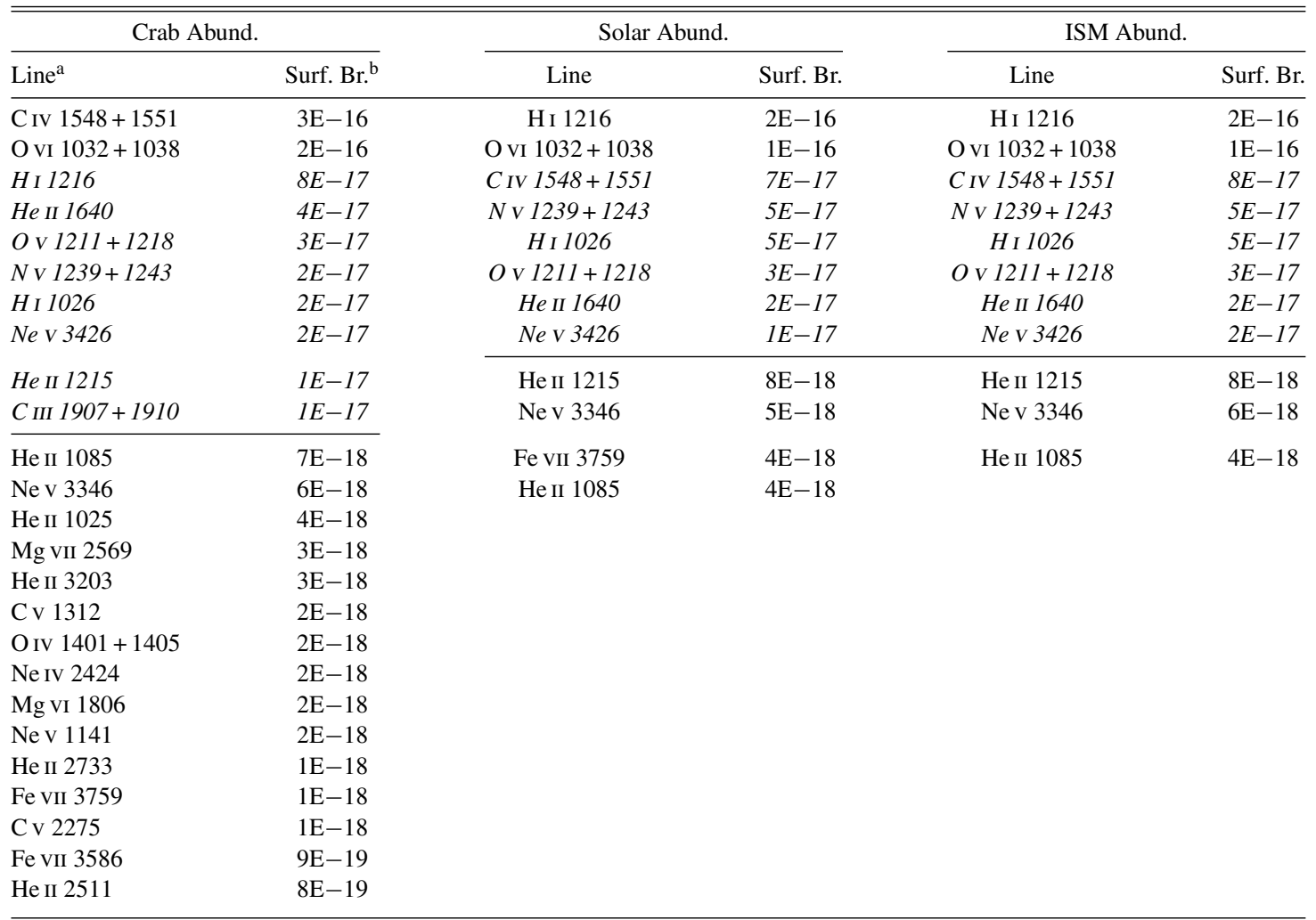

Notes. Italicized entries have predicted surface brightness at or above the current optical-passband detection limit.

a Wavelengths are given in $\AA$ unless noted with $\mathrm{m}=$ microns.

b Surface brightness, erg cm $\mathrm{cm}^{-2} \mathrm{~s}^{-1} \operatorname{arcsec}^{-2}$. 


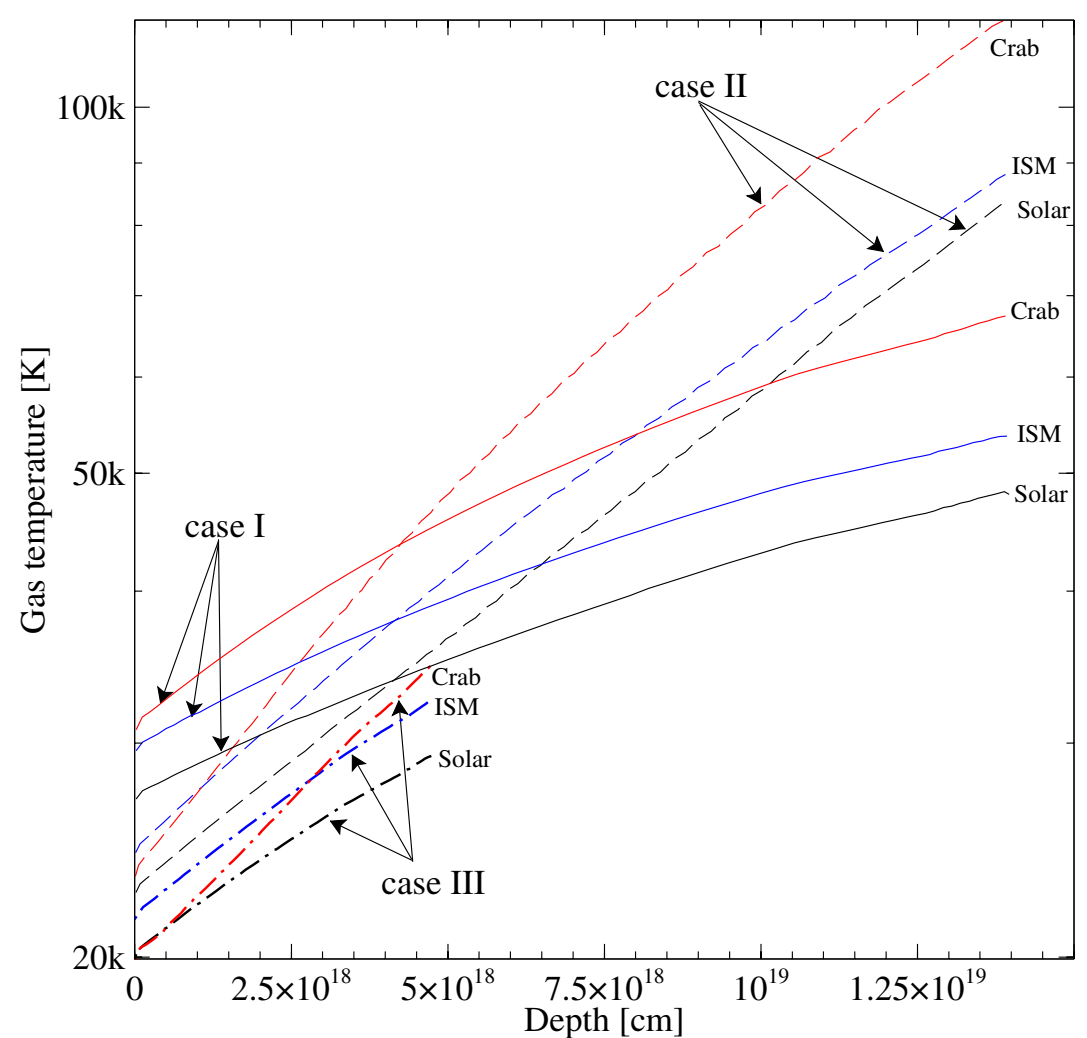

Figure 4. Gas temperature across the Crab outer shell for all the models. The depth is the distance between the illuminated face of the outer shell and a point within the outer shell.

(A color version of this figure is available in the online journal.)

Table 8

Predicted IR Emission Line Average Surface Brightness, Sorted by Surface Brightness for Each Model, for All Lines Brighter than H $\beta$, for Case III

\begin{tabular}{|c|c|c|c|c|c|}
\hline \multicolumn{2}{|c|}{ Crab Abund. } & \multicolumn{2}{|c|}{ Solar Abund. } & \multicolumn{2}{|c|}{ ISM Abund. } \\
\hline Line $^{\mathrm{a}}$ & Surf. Br. ${ }^{b}$ & Line & Surf. Br. & Line & Surf. Br. \\
\hline$[\mathrm{Ne}$ VI] $7.652 \mathrm{~m}$ & $1 E-16$ & {$[\mathrm{Ne}$ VI] $7.652 \mathrm{~m}$} & $8 E-17$ & {$[\mathrm{Ne}$ VI] $7.652 \mathrm{~m}$} & $1 E-16$ \\
\hline$[\mathrm{Ne} v] 24.31 \mathrm{~m}$ & $7 E-17$ & {$[\mathrm{Ne} \mathrm{V}] 24.31 \mathrm{~m}$} & $5 E-17$ & {$[\mathrm{Ne} \mathrm{v}] 24.31 \mathrm{~m}$} & $6 E-17$ \\
\hline$[\mathrm{Ne}$ V] $14.32 \mathrm{~m}$ & $5 E-17$ & {$\left[O_{I V}\right] 25.88 m$} & $5 E-17$ & {$[\mathrm{Ne}$ v] $14.32 \mathrm{~m}$} & $5 E-17$ \\
\hline$\left[O_{I V}\right] 25.88 m$ & $3 E-17$ & {$[\mathrm{Ne} \mathrm{V}] 14.32 \mathrm{~m}$} & $4 E-17$ & {$\left[O_{I V}\right] 25.88 \mathrm{~m}$} & $3 E-17$ \\
\hline$[\mathrm{Mg}$ VII] $9.033 \mathrm{~m}$ & $8 \mathrm{E}-18$ & [Fe VII] $9.508 \mathrm{~m}$ & $2 E-17$ & & \\
\hline$[\mathrm{Mg}$ VII $] 5.503 \mathrm{~m}$ & $7 E-18$ & {$[\mathrm{Mg}$ VII] $9.033 \mathrm{~m}$} & $1 E-17$ & & \\
\hline$[\mathrm{Fe}$ VII $] 9.508 \mathrm{~m}$ & $6 \mathrm{E}-18$ & [Si VII] $2.481 \mathrm{~m}$ & $1 E-17$ & & \\
\hline [He II $] 1.012 \mathrm{~m}$ & $4 \mathrm{E}-18$ & & & & \\
\hline$[\mathrm{Mg}$ VIII] $3.030 \mathrm{~m}$ & $3 \mathrm{E}-18$ & & & & \\
\hline [Si VII] $2.481 \mathrm{~m}$ & $3 E-18$ & & & & \\
\hline
\end{tabular}

Notes. Italicized entries have predicted surface brightness at or above the current optical-passband detection limit.

${ }^{a}$ Wavelengths are given in $\AA$ unless noted with $\mathrm{m}=$ microns.

${ }^{\mathrm{b}}$ Surface brightness, erg cm${ }^{-2} \mathrm{~s}^{-1} \operatorname{arcsec}^{-2}$.

where $n_{e}$ is the electron density and $\alpha_{\mathrm{B}}\left(T_{e}\right)$ is the Case B recombination coefficient at temperature $T_{e}$. The gas in the outer shell is photoionized by light from the visible Crab. Since Cloudy supposes that the gas atomic processes that are responsible for thermal and ionization equilibrium have reached steady state, we need to compare the age of the Crab with the recombination time to see if this is valid. We compute the recombination timescale for $\mathrm{Ne}^{+6} \rightarrow \mathrm{Ne}^{+5}$ for all three cases with solar abundances. We focus on this ion since it produces the strongest IR line. Since the temperature increases very slowly but the electron density decreases very quickly, we assume different radii have roughly the same recombination coefficient and evaluate it from the Badnell (2006), Badnell et al. (2003), and Badnell
Web site (http://amdpp.phys.strath.ac.uk/tamoc/DR/). We find the recombination times are about $100 \mathrm{yr}, 20 \mathrm{yr}$, and $10 \mathrm{yr}$ in the inner edge of the shell for cases I, II, and III, respectively (Figure 5). All of these are much shorter than the age of the visible Crab, suggesting that the outer shell has reached photoionization equilibrium.

\subsubsection{Thermal Timescale}

We calculate both the thermal energy $\left(\mathrm{erg} \mathrm{cm}^{-3}\right)$ and the cooling rate $\left(\mathrm{erg} \mathrm{cm}^{-3} \mathrm{~s}^{-1}\right.$ ) as a function of the radius for all three cases with solar abundances. From the ratio we can find the cooling time. We also calculate the emission measure for different radii or different zones. The differential emission 
Table 9

Predicted Optical Emission Line Average Surface Brightness, Sorted by Surface Brightness for Each Model, for All Lines Brighter than $\mathrm{H} \beta$, for Case III

\begin{tabular}{|c|c|c|c|c|c|}
\hline \multicolumn{2}{|c|}{ Crab Abund. } & \multicolumn{2}{|c|}{ Solar Abund. } & \multicolumn{2}{|c|}{ ISM Abund. } \\
\hline Line $^{a}$ & Surf. Br. ${ }^{b}$ & Line & Surf. Br. & Line & Surf. Br. \\
\hline He II 4686 & $2 E-17$ & H I 6563 & $2 E-17$ & H I 6563 & $2 E-17$ \\
\hline Н г 6563 & $6 \mathrm{E}-18$ & Fe VII 6087 & $2 E-17$ & Н г 4861 & $8 \mathrm{E}-18$ \\
\hline Fe VII 6087 & $5 E-18$ & Fe VII 5721 & $1 E-17$ & & \\
\hline Fe VII 5721 & $3 \mathrm{E}-18$ & Н г 4861 & $9 \mathrm{E}-18$ & & \\
\hline Н I 4861 & $2 \mathrm{E}-18$ & & & & \\
\hline
\end{tabular}

Notes. Italicized entries have predicted surface brightness at or above the current optical-passband detection limit.

${ }^{\text {a }}$ Wavelengths are given in $\AA$ unless noted with $\mathrm{m}=$ microns.

b Surface brightness, erg cm ${ }^{-2} \mathrm{~s}^{-1} \operatorname{arcsec}^{-2}$.

measure for each depth is then

$$
d \mathrm{EM}=4 \pi r^{2} n(r)^{2} d r .
$$

This gives an indication of which portions of the shell contribute most to the observed emission.

Figure 6 shows the cooling times and the differential emission measure across the Crab outer shell for all three cases with solar abundances. We find the cooling time for all cases to be much longer than the age of the visible Crab. Even for the inner edge, which produces much of the emission measure, the cooling times are still about 20,10, and 6 times of the age of the visible

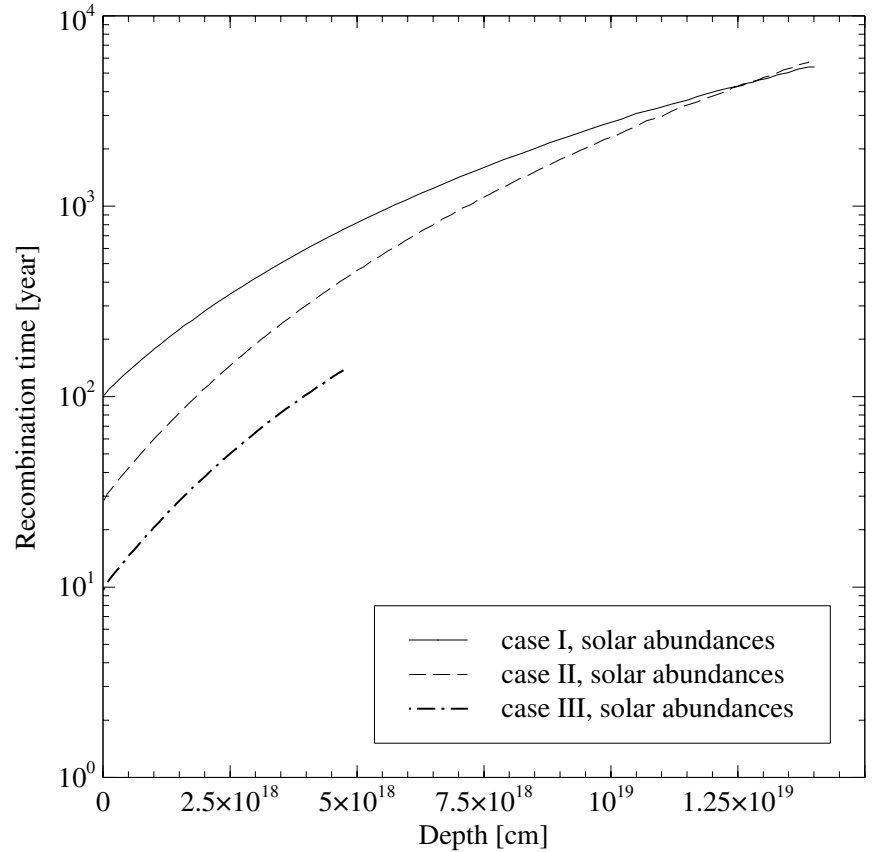

Figure 5. Recombination timescales for producing $\mathrm{Ne}^{+5}$ as a function of depth in the Crab outer shell for all three cases with solar abundances.

Crab for cases I, II, and III, respectively. This indicates that the outer shell has not had time to reach thermal equilibrium, so it retains a memory of its temperature in the past.

Table 10

Predicted UV Emission Line Average Surface Brightness, Sorted by Surface Brightness for Each Model, for All Lines Brighter than H $\beta$, for Case III

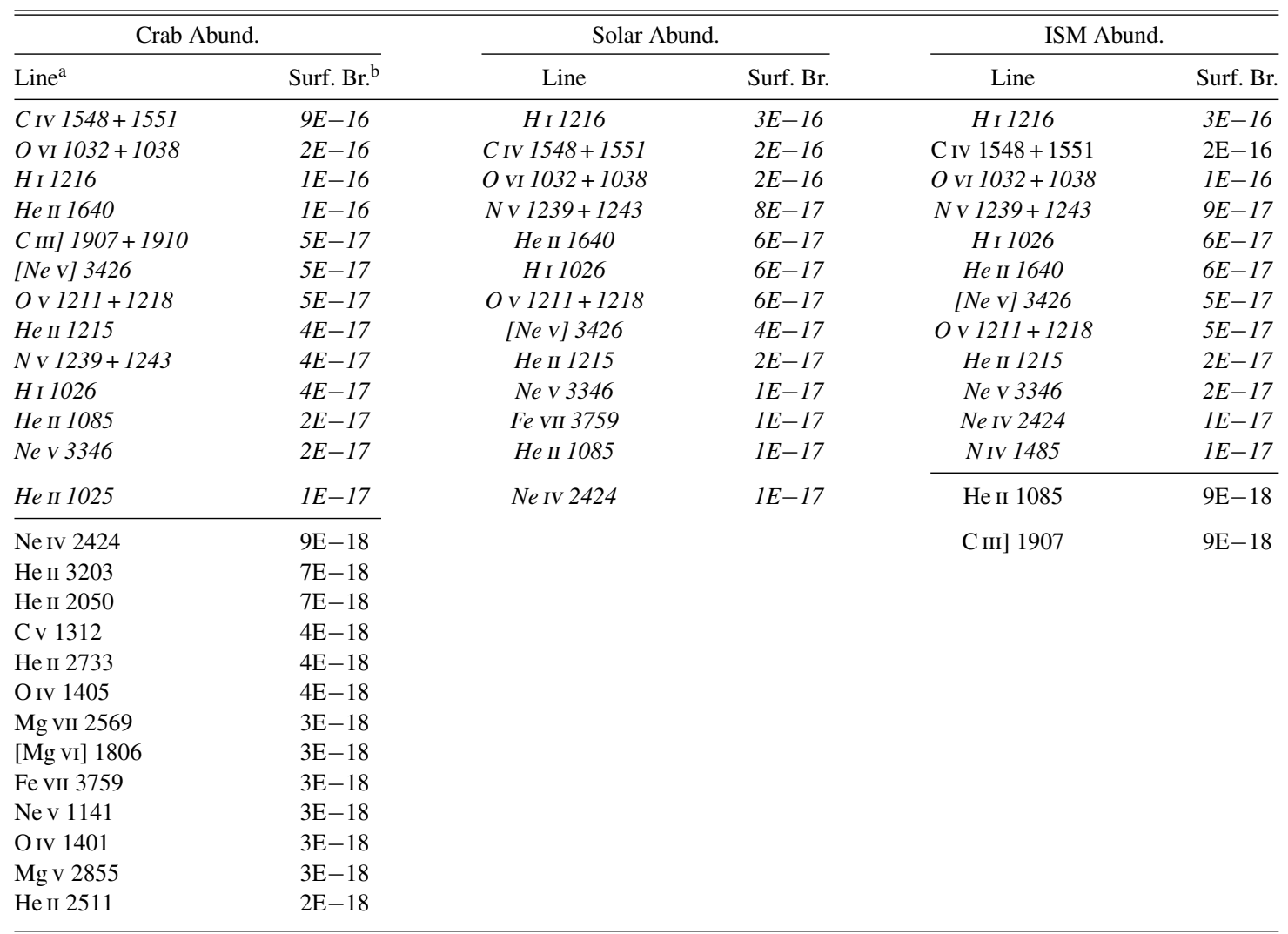

Notes. Italicized entries have predicted surface brightness at or above the current optical-passband detection limit.

a Wavelengths are given in $\AA$ unless noted with $\mathrm{m}=$ microns.

b Surface brightness, erg cm ${ }^{-2} \mathrm{~s}^{-1} \operatorname{arcsec}^{-2}$. 

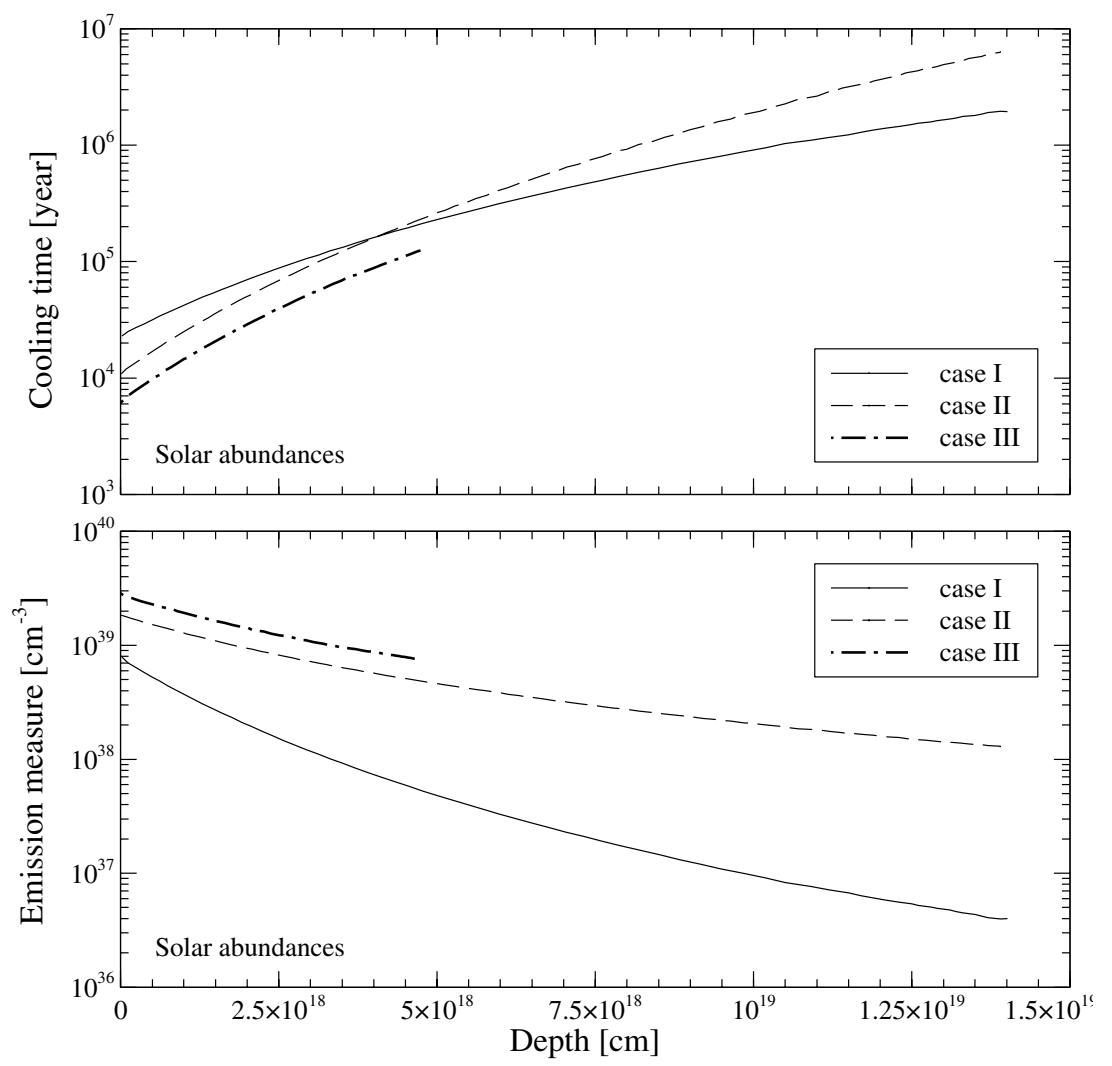

Figure 6. Cooling times and differential emission measures for all three cases with solar abundances. The innermost regions have the greatest emission measure and so would contribute the most to the observed spectrum.

Table 11

H I Luminosities for Different Cases (Case I, Solar) [erg s ${ }^{-1}$ ]

\begin{tabular}{lccc}
\hline \hline Line & Total & Case B & Case A \\
\hline Н г $\lambda 6563$ & $2.09 \mathrm{E}+32$ & $1.75 \mathrm{E}+32$ & $1.04 \mathrm{E}+32$ \\
Н $\lambda$ I 4861 & $7.31 \mathrm{E}+31$ & $6.47 \mathrm{E}+31$ & $4.02 \mathrm{E}+31$ \\
Н $\lambda 1216$ & $5.86 \mathrm{E}+33$ & $2.37 \mathrm{E}+33$ & $1.40 \mathrm{E}+33$ \\
Н $1.875 \mu \mathrm{m}$ & $2.05 \mathrm{E}+31$ & $1.68 \mathrm{E}+31$ & $1.43 \mathrm{E}+31$ \\
\hline
\end{tabular}

\subsubsection{Effects on Predicted Spectrum}

The shell is in photoionization, but not thermal, equilibrium. This means that atomic processes that set the ionization of the gas have reached steady state, and that the predicted ionization should be accurate. The fact that the gas is not in thermal equilibrium means that we do not really know its temperature, only that it is young enough to "remember" its temperature long ago. In other words, the current temperature is partially determined by its temperature in the past. We do not know whether the outer shell was initially hot or cold.

All of this is important because we predict that high ionization IR lines should be among the strongest lines in the optical-IR spectrum. Are these predictions approximately valid? The uncertain temperature should not greatly affect the high ionization lines in the IR. The emissivities of an IR collisionally excited line do not have a strong temperature dependence. Because the lines have low excitation potentials, their Boltzmann factors should be close to unity, so their emissivity is proportional to $T^{-1 / 2}$ (AGN3). The optical recombination lines have an emissivity that is a faster power law, typically $T^{-0.8}$. Factors of two uncertainties in the temperature carry over to uncertainties in the line's surface brightness by well less than a factor of two.
Similarly, the uncertain temperature should not greatly affect the predicted ionization of the gas. The ionization is set by the photoionization and recombination rates. The photoionization rate has no temperature dependence, while recombination coefficients have power-law temperature dependencies, roughly $T^{-0.8}$. Factors of two uncertainties in the temperature will change the ionization by less than this.

Lundqvist et al. (1986) gave time-dependent numerical simulations. We do have the ability to do time dependent, fully advective, photoionization flows (Henney et al. 2005, 2007). However, these calculations would have to be guided by observations that do not now exist. Is the shell cooling down from a hotter phase, warming up from a colder phase, or is it now in approximate thermal equilibrium?

\section{DISCUSSION AND CONCLUSIONS}

We have presented a series of photoionization equilibrium calculations of the properties of the outer shell in the Crab Nebula. We reached the following conclusions.

1. The gas cooling time is far longer than the age of the visible $\mathrm{Crab}$, so the outer shell is not in thermal equilibrium. As a result, we do not really know its temperature since it will carry a memory of its original value.

2. The recombination time is much shorter than the age of the Crab, so the outer shell is in ionization equilibrium.

3. Together these mean that the outer shell will be highly ionized but we are not certain of its temperature. We find that the IR coronal lines are very strong, stronger than most optical lines used in previous searches. Luckily, these lines are not sensitive to the gas temperature so this is a robust prediction. 
Table 12

Predicted Optical Depth, Sorted by Wavelength for Thermal-broadened Model, for Case I

\begin{tabular}{|c|c|c|c|c|c|}
\hline \multicolumn{2}{|c|}{ Crab Abund. } & \multicolumn{2}{|c|}{ Solar Abund. } & \multicolumn{2}{|c|}{ ISM Abund. } \\
\hline Line $^{a}$ & Opt. Dpt. ${ }^{\mathrm{b}}$ & Line & Opt. Dpt. & Line & Opt. Dpt. \\
\hline O I 1025 & $3.56 \mathrm{E}-01$ & Or 1025 & $1.62 \mathrm{E}+00$ & Oі 1025 & $1.42 \mathrm{E}+00$ \\
\hline НІ 1025 & $4.05 \mathrm{E}-01$ & Нг 1025 & $1.88 \mathrm{E}+00$ & Hг 1025 & $1.63 E+00$ \\
\hline O VI $1031+1037$ & $3.21 \mathrm{E}+01$ & O vi $1031+1037$ & $3.81 \mathrm{E}+01$ & O vi $1031+1037$ & $2.19 \mathrm{E}+01$ \\
\hline Н I 1215 & $2.53 E+00$ & Н I 1215 & $1.17 \mathrm{E}+01$ & Н I 1215 & $1.02 \mathrm{E}+01$ \\
\hline Nv $1239+1243$ & $1.03 \mathrm{E}+00$ & $\mathrm{Nv} 1239+1243$ & $3.54 \mathrm{E}+00$ & $\mathrm{Nv} 1239+1243$ & $2.88 \mathrm{E}+00$ \\
\hline C IV $1548+1551$ & $1.32 \mathrm{E}+01$ & Civ $1548+1551$ & $2.68 \mathrm{E}+00$ & Civ $1548+1551$ & $2.33 \mathrm{E}+00$ \\
\hline
\end{tabular}

Notes.

a Wavelengths are given in $\AA$.

${ }^{\mathrm{b}}$ Optical depth.

Table 13

Predicted Optical Depth, Sorted by Wavelength for Thermal-broadened Model, for Case II

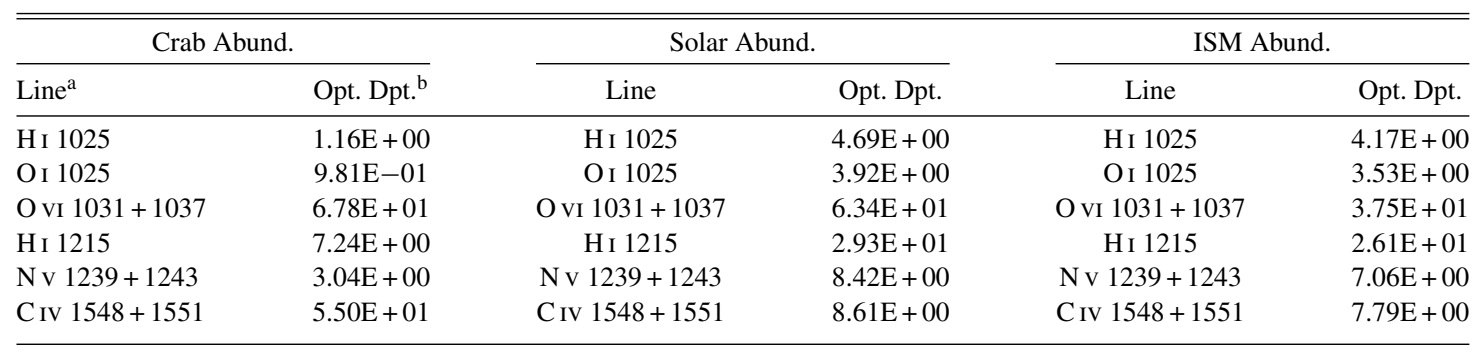

Notes.

a Wavelengths are given in $\AA$.

${ }^{\mathrm{b}}$ Optical depth.

Table 14

Predicted Optical Depth, Sorted by Wavelength for Thermal-broadened Model, for Case III

\begin{tabular}{|c|c|c|c|c|c|}
\hline \multicolumn{2}{|c|}{ Crab Abund. } & \multicolumn{2}{|c|}{ Solar Abund. } & \multicolumn{2}{|c|}{ ISM Abund. } \\
\hline Or 1025 & $2.86 \mathrm{E}+00$ & OI 1025 & $1.06 \mathrm{E}+01$ & H I 1025 & $1.18 \mathrm{E}+01$ \\
\hline O vi $1031+1037$ & $1.34 \mathrm{E}+02$ & O VI $1031+1037$ & $1.03 \mathrm{E}+02$ & O vi $1031+1037$ & $6.24 \mathrm{E}+01$ \\
\hline H I 1215 & $2.19 \mathrm{E}+01$ & H I 1215 & $8.17 E+01$ & H I 1215 & $7.37 \mathrm{E}+01$ \\
\hline \multirow[t]{2}{*}{ C IV $1548+1551$} & $2.00 \mathrm{E}+02$ & Si IV 1394 & $1.47 \mathrm{E}-01$ & C IV $1548+1551$ & $2.51 \mathrm{E}+01$ \\
\hline & & C IV $1548+1551$ & $2.72 \mathrm{E}+01$ & & \\
\hline
\end{tabular}

Notes.

a Wavelengths are given in $\AA$.

b Optical depth.

Table 15

Line Optical Depths for Static and Dynamic Cases (Case I, Solar)

\begin{tabular}{lcc}
\hline \hline Line & Thermal & $v=1680 \mathrm{~km} \mathrm{~s}^{-1}$ \\
\hline O VI $\lambda 1031+\lambda 1037$ & 38.1 & $1.81 \mathrm{E}-01$ \\
C IV $\lambda 1548+\lambda 1551$ & 2.68 & $1.95 \mathrm{E}-02$ \\
\hline
\end{tabular}

4. The outer shell can produce the observed C IV absorption if the line broadening across the line-forming region is not large. Full dynamical solutions would be needed to make robust predictions of this line optical depth.

5. The existing observational limit on $\mathrm{H} \alpha$ does not place useful constraints on most of our models, but it is on the verge of ruling out models with solar and ISM abundances, $\alpha<-4$, $v \propto r^{2}$, and that contain the full amount of the missing mass.
6. The IR coronal lines are our best hope for avoiding confusion with scattered light from the inner parts of the Crab. The species producing them are too highly ionized to be produced by the photoionized gas in the filaments, and are higher ionization than the shocked gas that directly produces the $[\mathrm{O} \mathrm{III}]$ emission skin at the outer edge of the synchrotron bubble (although higher velocity shocks could co-exist in this latter region and produce such lines).

7. We recommend imaging (or spectroscopy) on the sky just outside the main part of the Crab to search for one of these IR lines.

8. An alternative approach would be to search for these lines in the spectra of the center of the Crab where the projected expansion velocities are toward and away from us.

We thank Peter Lundqvist for his careful review of our manuscript. G.J.F. acknowledges support by NSF (1108928; and 
1109061), NASA (10-ATP10-0053, 10-ADAP10-0073, and NNX12AH73G), and STScI (HST-AR-12125.01, GO-12560, and HST-GO-12309). J.A.B. and C.T.R. acknowledge support by NSF (1006593). C.T.R., J.A.B., and E.D.L. are grateful to NASA for support through ADAP grant NNX10AC93G.

\section{REFERENCES}

Badnell, N. R. 2006, ApJS, 167, 334

Badnell, N. R., O'Mullane, M. G., Summers, H. P., et al. 2003, A\&A, 406, 1151 Chevalier, R. A. 1977, in Supernovae, ed. D. Schramm (Astrophysics and Space Science Library, Vol. 66; Berlin: Springer), 53

Davidson, K., \& Fesen, R. A. 1985, ARA\&A, 23, 199

Ferland, G. J. 1980, PASP, 92, 596

Ferland, G. J., Porter, R. L., van Hoof, P. A. M., et al. 2013, RevMexAA, 49, 137 (F13)

Fesen, R. A., Shull, J. M., \& Hurford, A. P. 1997, AJ, 113, 354

Henney, W. J., Arthur, S. J., Williams, R. J. R., \& Ferland, G. J. 2005, ApJ, 621,328
Henney, W. J., Williams, R. J. R., Ferland, G. J., Shaw, G., \& O’Dell, C. R. 2007, ApJ, 671, 137

Hester, J. J. 2008, ARA\&A, 46, 127

Kitaura, F. S., Janka, H.-T., \& Hillebrandt, W. 2006, A\&A, 450, 345

Lundqvist, P., Fransson, C., \& Chevalier, R. A. 1986, A\&A, 162, L6

Lundqvist, P., \& Tziamtzis, A. 2012, MNRAS, 423, 1571

Nomoto, K. 1985, in The Crab Nebula and Related Supernova Remnants, ed. M. C. Kafatos \& R. B. C. Henry (Cambridge: Cambridge Univ. Press), 97

Nomoto, K. 1987, ApJ, 322, 206

Osterbrock, D. E., \& Ferland, G. J. 2006, Astrophysics of Gaseous Nebulae and Active Galactic Nuclei (Mill Valley, CA: Univ. Science Books)

Pequignot, D., \& Dennefeld, M. 1983, A\&A, 120, 249

Sankrit, R., \& Hester, J. J. 1997, ApJ, 491, 796

Smith, N. 2013, MNRAS

Sollerman, J., Lundqvist, P., Lindler, D., et al. 2000, ApJ, 537, 861

Storey, P. J., \& Hummer, D. G. 1995, MNRAS, 272, 41

Temim, T., Sonneborn, G., Dwek, E., et al. 2012, ApJ, 753, 72

Trimble, V. L. 1968, AJ, 73, 535

Tziamtzis, A., Schirmer, M., Lundqvist, P., \& Sollerman, J. 2009, A\&A, 497, 167 